\title{
The implications of low quality bicycle paths on gaze behavior of cyclists : a field test
}

Department of Movement and Sports Sciences, Ghent University, Belgium

\section{Introduction}

For a bicyclist to participate safely in busy traffic the ability to foresee events and hazards is vital (Hosking et al. 2010). Therefore, it is important that the bicycle environment allows a shift of attention from the 'personal space' towards traffic events and other traffic users. One factor that might demand a significant proportion of the attention capacity is the quality of the cycling tracks. The presence of irregularities in the surface might impede the visual attention to shift towards the wide environment (Pelz, 2007), resulting in wrong or late decisions that might affect the safety of bicycle users.

The aim of this experiment was to investigate to what extent the cycle track surface quality affects visual attention as measured by eye tracking in adult bicycle users.

\section{Methods}

Twelve adults (22 to 24 years of age) who use their bicycle on a daily basis took part in the study and completed the four $\mathbf{k m}$ cycling tour in Ghent wearing an eye tracker. All participants had normal or corrected-to normal vision. Since direct sunlight disturbed the infra red signal of the eye tracking system, all tests were performed in overcast but dry weather.

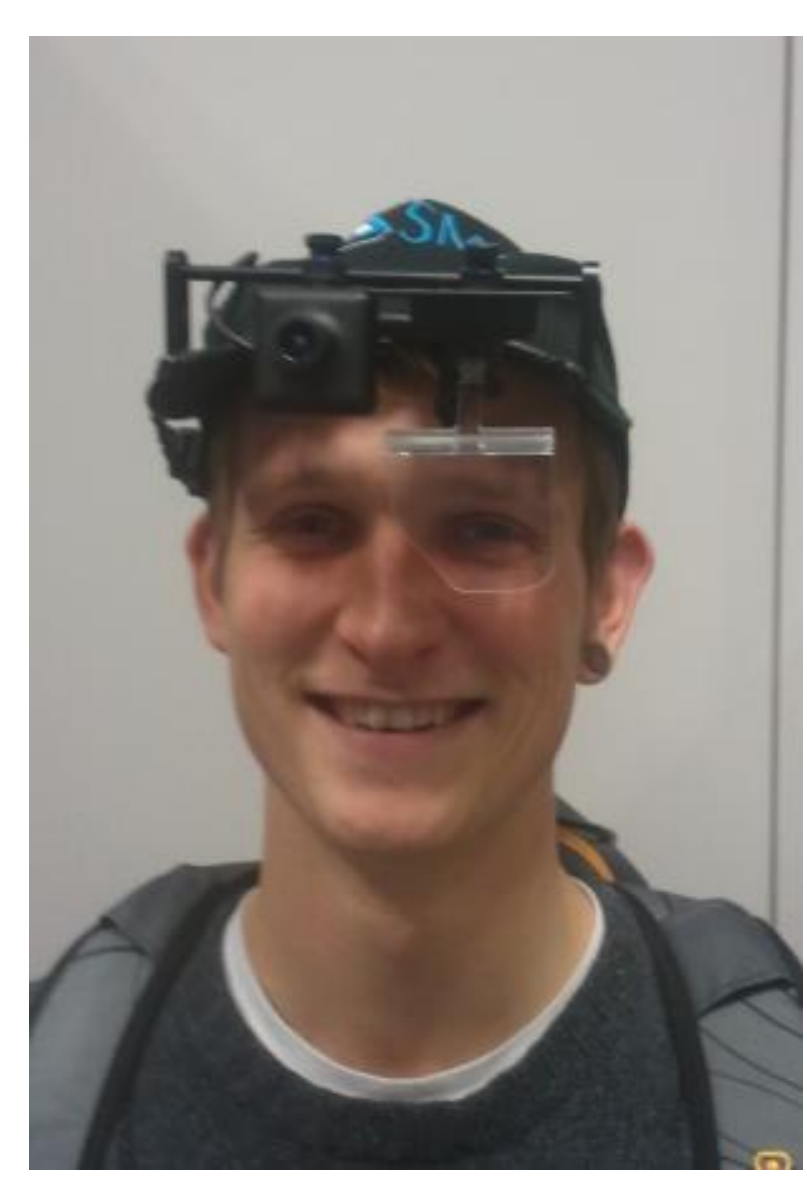

Figure 1 : Participant wearing a head mounted eye tracker The system (HED, SMI, Germany), with a $1^{\circ}$ accuracy was mounted on a baseball cap and recorded the left eye movements with an infrared-sensitive camera at $50 \mathrm{~Hz}$ using pupil position and corneal reflex. Scene video was recorded at $25 \mathrm{~Hz}$, placed next to the eye-tracking camera. The two cameras were connected to a notebook which was worn in a backpack.

After the tests, five participants were selected for further analysis, based on following inclusion criteria:

Good Pre- and Post- Calibration of eye tracker

Percentage of No Data (due to saccades, blinking, gazing outside reference frame, interference of sunlight or loss of pupil/corneal reflex data) did not exceed $30 \%$

- Not being obstructed by other cyclists

A $120 \mathrm{~m}$ long trial was selected on both tracks for analysis (Thick red/green part in Fig. 2). Videos with gaze cursor were coded frame by frame to assign gaze location to one of following Areas Of Interest (AOI): Road, Side, External, Focus of Expansion and Cyclist. When no data was available the frame was coded as 'NoData'.
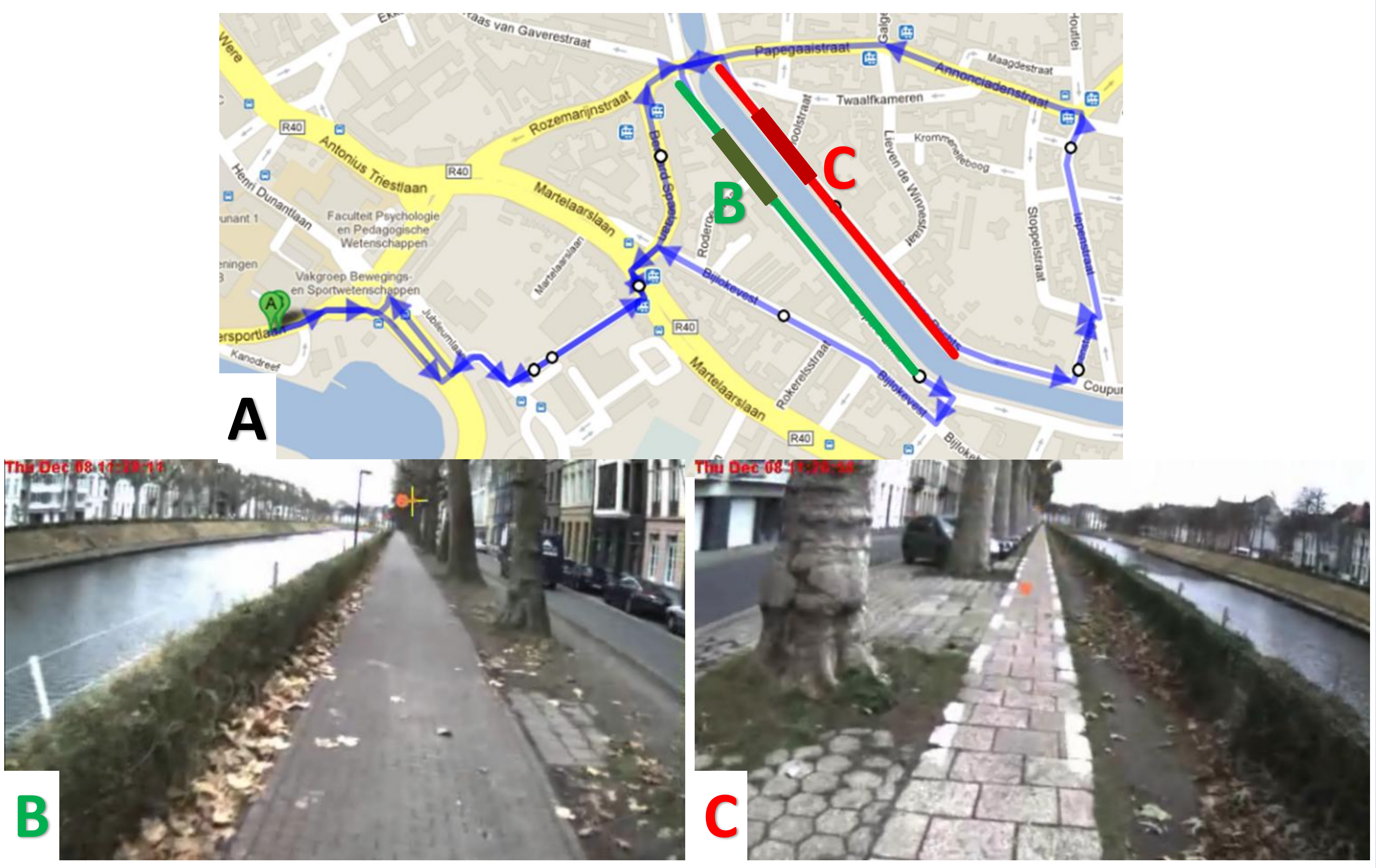

Figure 2 : the cycling route $(\mathbf{A})$ included two bicycle tracks with approximately the same width and similar environment but with different surfaces. The High Quality track (B) was recently renovated and had a brick surface; the Low Quality track (C) had a surface of large tiles that showed several deformities and cracks. Both tracks were physically separated from the carriageway by trees and/or parked cars.
Corresponding author: pieter.vansteenkiste@ugent.be

\section{Results}

On the Low Quality track, participants looked significantly more to the road. No difference was found in looking to the Focus of Expansion. All other regions were gazed at less on the Low than on the High Quality track.

\begin{tabular}{|c|c|c|c|c|c|c|c|c|c|c|c|c|}
\hline$\%$ & & & High & Quality & & & & & Lon & Quality & & \\
\hline Subj. & Road & FoE & Side & Extern & Cyclist & $\begin{array}{l}\text { No } \\
\text { Data }\end{array}$ & Road & FoE & Side & Extern & Cyclist & $\begin{array}{l}\text { No } \\
\text { Data }\end{array}$ \\
\hline CM & 21 & 0 & 19 & 16 & 25 & 19 & 90 & 1 & 2 & 0 & 0 & 7 \\
\hline GM & 10 & 7 & 22 & 24 & 12 & 25 & 40 & 0 & 32 & 7 & 0 & 21 \\
\hline I L & 03 & 26 & 21 & 26 & 6 & 17 & 35 & 24 & 4 & 27 & 0 & 11 \\
\hline IV & 28 & 6 & 21 & 30 & 6 & 10 & 76 & 0 & 4 & 9 & 0 & 10 \\
\hline LM & 62 & 5 & 3 & 5 & 16 & 10 & 73 & 12 & 3 & 0 & 7 & 6 \\
\hline & $* *$ & & & * & $* *$ & * & $* *$ & & & * & $* *$ & * \\
\hline & $\mathrm{Q}$ & & & & & & & & & & & \\
\hline & 1Q & & & & & & & & & & & \\
\hline & $0 \%$ & & & & 40 & & 60 & & & & & $\%$ \\
\hline
\end{tabular}

Figure 3 : Percentage of gaze towards Area's Of Interest for each participant $\left.{ }^{* *}: p<0.05 ;{ }^{*}: 0.1>p>0.05\right)$ and Average Percentages of gaze to each Area of Interest. LQ = Low Quality Track; HQ = High Quality track

\section{Conclusions}

Due to the low quality of the bicycle path, the cycling task itself became more demanding, which resulted in a shift of attention from distant environmental regions to more proximate road properties. Therefore low quality bicycle tracks may affect the alertness and responsiveness of cyclists to environmental hazards. These results confirm the importance of qualitative cycling infrastructure.

In addition, results of current study were in line with the model for gaze behavior during goal directed locomotion as proposed by Vansteenkiste et al. (in press) .

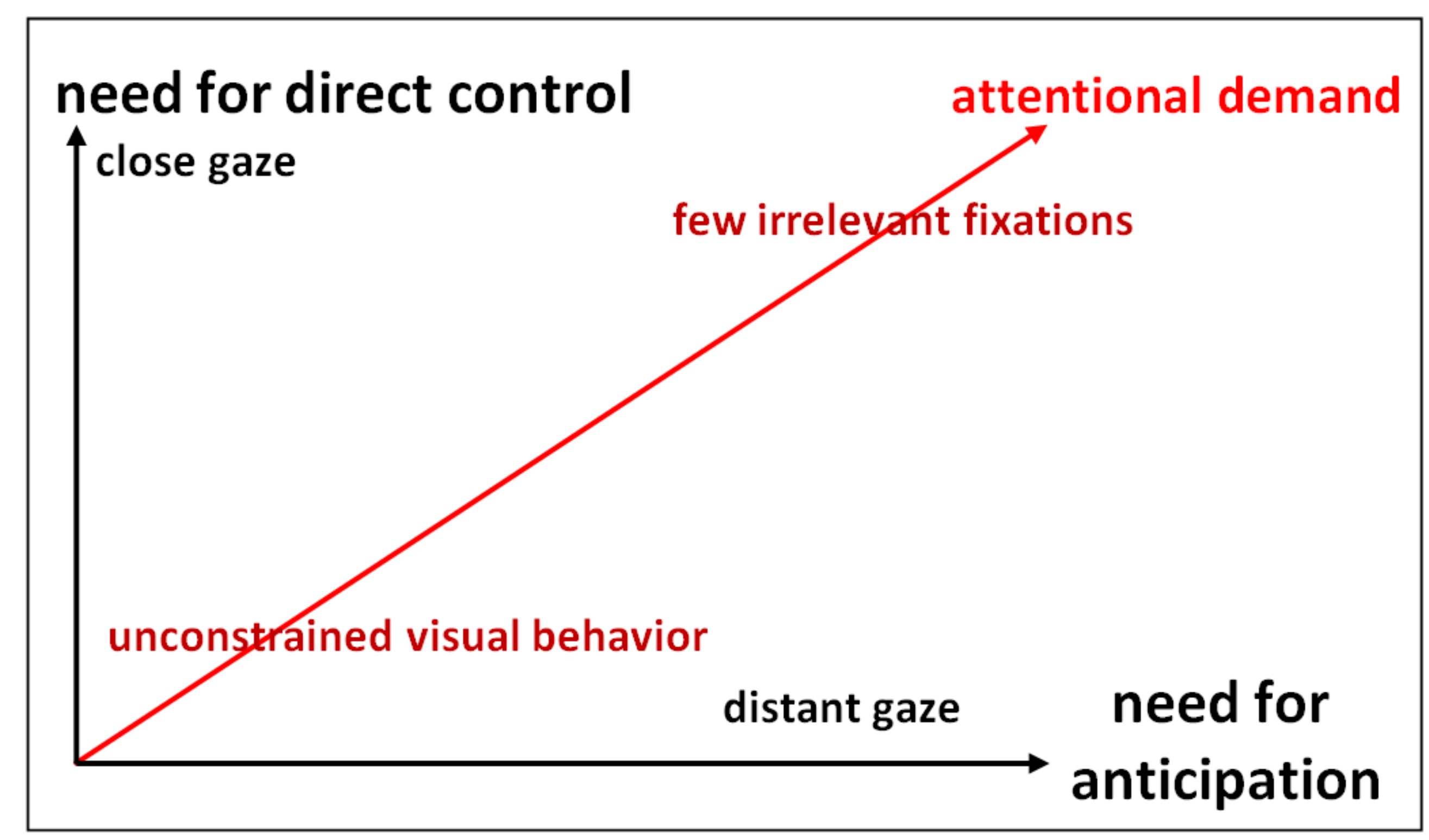

Figure 5 : model for gaze behavior during goal directed locomotion. Direction o gaze is determined by the need for direct control and the need for anticipation. When both are high, the attentional demand will be high as well.

\section{References}

Hosking, S. G., Liu, C. C., \& Bayly, M. (2010). The visual search patterns and hazard responses of experienced and inexperienced motorcycle riders. Accident Analysis and Prevention, 42, 196-202.

Pelz, J. B. (2007). Oculomotor behavior in natural and man-made environments. Eye Movements: a window on Mind and Brain (pp. 661-676).

Vansteenkiste, P., Cardon, G., D'Hondt, E., Philippaerts, R., Lenoir, M. (2012) The visual control of bicycle steering: the effects of speed and path width. Accident Analysis and Prevention, In Press

\section{Acknowledgements}

This research was supported by the life line campaign of the Research Foundation of Flanders (FWO) FWO G.A115.11N. Special thanks go to Ilse Picavet and Fran Ketels for helping with data collection and analysis. 